\title{
The Onset of Buoyancy and Surface Tension Driven Convection in a Ferrofluid Layer by Influence of General Boundary Conditions
}

\author{
Mahesh Kumar Ramachandraiah1, Savitha Basavaraju² \\ ${ }^{1}$ MES Pre-University College of Arts, Commerce and Science, Bengaluru, India \\ ${ }^{2}$ Raja Rajeswari College of Engineering, Bengaluru, India \\ Email: rmaheshkumar78@rediffmail.com,savibraj@gmail.com
}

How to cite this paper: Ramachandraiah, M.K. and Basavaraju, S. (2022) The Onset of Buoyancy and Surface Tension Driven Convection in a Ferrofluid Layer by Influence of General Boundary Conditions. Open Journal of Fluid Dynamics, 12, 56-68. https://doi.org/10.4236/ojfd.2022.121003

Received: January 2, 2022

Accepted: February 27, 2022

Published: March 2, 2022

Copyright $\odot 2022$ by author(s) and Scientific Research Publishing Inc. This work is licensed under the Creative Commons Attribution International License (CC BY 4.0).

http://creativecommons.org/licenses/by/4.0/

\begin{abstract}
This paper investigated the buoyancy and surface tension-driven ferro-thermal-convection (FTC) in a ferrofluid (FF) layer due to influence of general boundary conditions. The lower surface is rigid with insulating to temperature perturbations, while the upper surface is stress-free and subjected to general thermal boundary condition. The numerically Galerkin technique (GT) and analytically regular perturbation technique (RPT) are applied for solving the problem of eigenvalue. It is analyzed that increasing Biot number, decreases the magnetic and Marangoni number is to postponement the onset. Additionally, magnetization nonlinearity parameter has no effect on FTC in the non-existence of Biot number. The results under the limiting cases are found to be in good agreement with those available in the literature.
\end{abstract}

\section{Keywords}

Marangoni Number, Ferrothermal Convection, Insulating, Regular Perturbation Technique, Galerkin Technique

\section{Introduction}

Until recently, there were liquids which could be magnetized to be comparable with the magnetization of magnetic nanoparticles. They have developed colloidal suspensions containing magnetic nanoparticles with a carrier liquid like water, hydrocarbon such as mineral oil or kerosene, or fluorocarbon referred as ferrofluids (FFs). Hence, FFs subjects have obtained much attention among the scientific communities [1] [2] [3] [4]. The magnetization of FFs depends on its magnetic field, temperature and density. Whereas when a horizontal FF layer is pre- 
sent with a magnetic field, it is heated from below and convective motions might take place which is called as FTC [5].

Thereby, FTC can also be induced by providing surface-tension and later with the function of temperature. Qin and Kaloni [6] have investigated both linear and non-linear stability of combined effects of buoyancy and surface tension forces in a FF layer. Hennenberg et al. [7] have examined the coupling effects on Marangoni and Rosensweig instabilities by considering two semi-infinite immiscible and incompressible viscous fluids. The results of different basic temperature gradients on FTC which is driven by buoyancy and surface tension forces discussed by Shivakumara et al. [8] with an plan following indulgent control of FTC concept. Shivakumara and Nanjundappa [9] have also examined the initiation of Marangoni FTC with differing initial temperature gradients. A very less number of researches address the effects of Bouyancy and surface tension forces on FTC (see [10] [11]) with viscosity variations ([12] [13] [14] [15]), heat source strength ([16] [17]) and Coriolis force ([18] [19] in a FF layer. Later, Shivakumara et al. [20] studied the onset of FTC in a horizontal FF layer with temperature dependent viscosity in exponentially. In many natural phenomena, the study of penetrative FTC in a saturated porous layer is studied by Nanjundappa et al. [21] with the internal heating source and applied Brinkman extended Darcy model in the momentum equation. Nanjundappa and co-workers ([22] [23] [24]) analyzed the internal heat generation effect on the onset of FTC in a FF saturated porous layer. Recently, Savitha et al. [25] investigated the penetrative FTC in a FF-saturated high porosity anisotropic porous layer via uniform internal heating.

The intent of the present work is to investigate Bénard-Marangoni FTC in a FF layer due to influence of general boundary conditions. The numerically Galerkin technique (GT) and analytically regular perturbation technique (RPT) are applied for solving the problem of eigenvalue when both the surfaces insulated to temperature perturbations.

\section{Formulation of the Problem}

Consider an incompressible FF horizontal layer of thickness $d$ with temperatures $-k_{1} \partial T / \partial z=q_{0} \quad(z=0)$ and $k_{1} \partial T / \partial z=h_{1}\left(T-T_{\infty}\right) \quad(z=d)$. Where $T$ is the temperature, $q_{0}$ is the conductive thermal flux, $k_{1}$ the overall thermal conductivity, $h_{t}$ the heat transfer coefficient and $T_{\infty}$ the temperature in the bulk of the environment.

Cartesian coordinates $(x, y, z)$ system are chosen (see Figure 1). Gravity acts vertically downwards and is given by $\vec{g}=-g \hat{k}$, where $\hat{k}$ is the unit vector in the z-direction. The layer is bounded below by a rigid surface and above by a non-deformable free surface. At the upper free surface, the surface tension $\sigma$ is assumed to vary linearly with temperature in the form

$$
\sigma=\sigma_{0}-\sigma_{T}\left(T-T_{0}\right)
$$




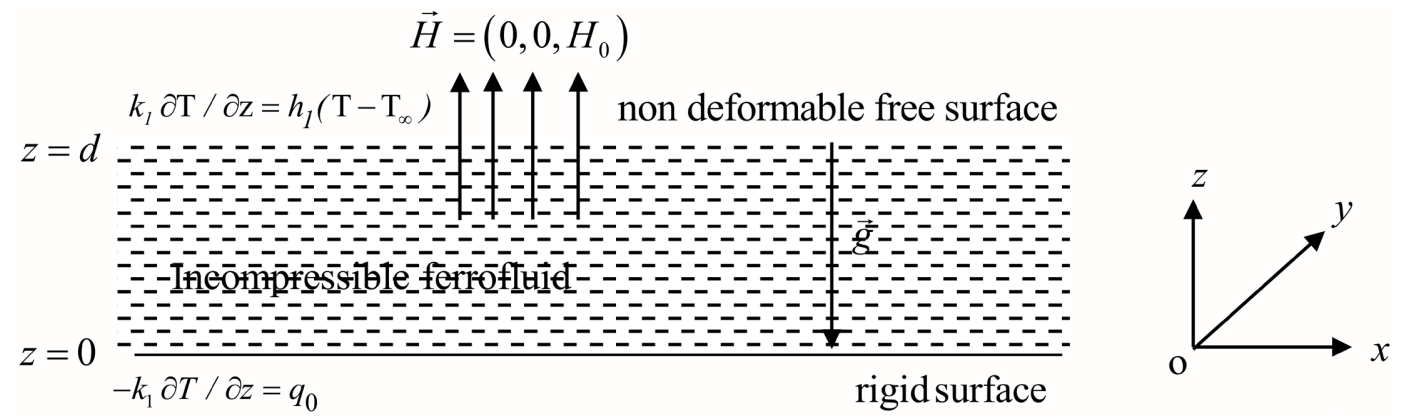

Figure 1. Physical configuration.

where $\sigma_{0}$ is the unperturbed value and $-\sigma_{T}$ is the rate of change of surface tension with temperature $T$. The fluid density $\rho$ is assume to vary linearly with temperature in the form

$$
\rho=\rho_{0}\left[1-\alpha_{t}\left(T-T_{0}\right)\right]
$$

where $\alpha_{t}$ is the thermal expansion coefficient and $\rho_{0}$ is the density at $T=T_{0}$. The governing equations for the flow of an incompressible fluid are

$$
\nabla \cdot \vec{q}=0
$$

where $\vec{q}=(u, v, w)$ is the velocity vector.

$$
\rho_{0}\left[\frac{\partial \vec{q}}{\partial t}+(\vec{q} \cdot \nabla) \vec{q}\right]=-\nabla p+\rho \vec{g}+\mu \nabla^{2} \vec{q}+\mu_{0}(\vec{M} \cdot \nabla) \vec{H}
$$

where $p$ is the pressure, $t$ is the time and $\mu_{0}$ the magnetic permeability of vacuum.

$$
\left[\rho_{0} C_{V, H}-\mu_{0} \vec{H} \cdot\left(\frac{\partial \vec{M}}{\partial T}\right)_{V, H}\right] \frac{D T}{D t}+\mu_{0} T\left(\frac{\partial \vec{M}}{\partial T}\right)_{V, H} \cdot \frac{D \vec{H}}{D t}=k_{t} \nabla^{2} T
$$

where $C$ is the specific heat, $C_{V, H}$ is the specific heat at constant volume and magnetic field, and $\nabla^{2}=\frac{\partial^{2}}{\partial x^{2}}+\frac{\partial^{2}}{\partial y^{2}}+\frac{\partial^{2}}{\partial z^{2}}$ is the Laplacian operator.

The magnetic field $(\vec{H})$ in magnetic fluid obeys the Maxwell equations in the absence electric field and current are

$$
\nabla \cdot \vec{B}=0, \nabla \times \vec{H}=0 \text { or } \vec{H}=\nabla \varphi
$$

where $\vec{B}$ is the magnetic induction and $\varphi$ is the magnetic potential.

$$
\vec{B}=\mu_{0}(\vec{M}+\vec{H})
$$

Since the magnetization $(\vec{M})$ depends on the magnitude of magnetic field and temperature, we have

$$
\vec{M}=\frac{\vec{H}}{H} M(H, T) .
$$

The linearized equation of magnetic state about $H_{0}$ and $T_{0}$ is

$$
M=M_{0}+\chi\left(H-H_{0}\right)-K\left(T-T_{0}\right)
$$

where $\chi=(\partial M / \partial H)_{H_{0}, T_{0}}$ is the magnetic susceptibility, $K=-(\partial M / \partial T)_{H_{0}, T_{0}}$ is 
the pyromagnetic co-efficient and $M_{0}=M\left(H_{0}, T_{0}\right)$.

It is clear that there exists the following solution for the basic state:

$$
\begin{gathered}
\vec{q}_{b}=0, \quad p_{b}(z)=p_{0}-\rho_{0} g z-\frac{1}{2} \rho_{0} \alpha_{t} g \beta z^{2}-\frac{\mu_{0} M_{0} \kappa \beta}{1+\chi} z-\frac{\mu_{0} \kappa^{2} \beta^{2}}{2(1+\chi)^{2}} z^{2} \\
T_{b}(z)=T_{0}-\beta z, \quad \vec{H}_{b}(z)=\left[H_{0}-\frac{K \beta z}{1+\chi}\right] \hat{k}, \quad \vec{M}_{b}(z)=\left[M_{0}+\frac{K \beta z}{1+\chi}\right] \hat{k}
\end{gathered}
$$

where $\beta=\Delta T / d$ is the temperature gradient and the subscript $b$ denotes the basic state.

To study the stability of the system, we perturb all the variables in the form

$$
\vec{q}=\vec{q}^{\prime}, \quad p=p_{b}(z)+p^{\prime}, T=T_{b}(z)+T^{\prime}, \vec{H}=\vec{H}_{b}(z)+\vec{H}^{\prime}, \vec{M}=\vec{M}_{b}(z)+\vec{M}^{\prime}
$$

where $\vec{q}^{\prime}, p^{\prime}, T^{\prime}, \vec{H}^{\prime}$ and $\vec{M}^{\prime}$ are perturbed variables and are assumed to be small.

Substituting Equation (11) into Equations (8) and (9), and using Equation (7), we obtain (after dropping the primes)

$$
\begin{aligned}
& H_{x}+M_{x}=\left(1+M_{0} / H_{0}\right) H_{x}, \\
& H_{y}+M_{y}=\left(1+M_{0} / H_{0}\right) H_{y}, \\
& H_{z}+M_{z}=(1+\chi) H_{z}-K T .
\end{aligned}
$$

Again substituting Equation (11) into momentum Equation (4), linearizing, eliminating the pressure term by operating curl twice and using Equation (12) the $\mathrm{z}$-component of the resulting equation can be obtained as (after dropping the primes):

$$
\left(\rho_{0} \frac{\partial}{\partial t}-\mu \nabla^{2}\right) \nabla^{2} w=-\mu_{0} K \beta \frac{\partial}{\partial z}\left(\nabla_{h}^{2} \varphi\right)+\frac{\mu_{0} K^{2} \beta}{1+\chi} \nabla_{h}^{2} T+\rho_{0} \alpha_{t} g \nabla_{h}^{2} T
$$

where $\nabla_{h}^{2}=\partial^{2} / \partial x^{2}+\partial^{2} / \partial y^{2}$ is the horizontal Laplacian operator. The temperature Equation (5), after using Equation (11) and linearizing, takes the form (after dropping the primes):

$$
\frac{\partial T}{\partial t}-\mu_{0} T_{0} K \frac{\partial}{\partial t}\left(\frac{\partial \varphi}{\partial z}\right)=k_{1} \nabla^{2} T+\left[\rho_{0} C_{0}-\frac{\mu_{0} T_{0} K^{2}}{1+\chi}\right] w \beta
$$

where $\rho_{0} C_{0}=\rho_{0} C_{V, H}+\mu_{0} H_{0} K$. Equations $6(\mathrm{a}, \mathrm{b})$, after substituting Equation (11) and using Equation (12), may be written as (after dropping the primes)

$$
\left(1+\frac{M_{0}}{H_{0}}\right) \nabla_{h}^{2} \varphi+(1+\chi) \frac{\partial^{2} \varphi}{\partial z^{2}}-K \frac{\partial T}{\partial z}=0 .
$$

The normal mode expansion of the dependent variables is assumed in the form

$$
\{w, T, \varphi\}=\{W(z), \Theta(z), \Phi(z)\} \exp [i(\omega t+\ell x+m y)]
$$

where $\ell$ and $m$ are wave numbers in the $x$ and $y$ directions, respectively, and $\omega$ is the growth rate with is complex. On substituting Equation (16) into Equations (13)-(15) and non-dimesionalizing the variables by setting 


$$
z^{*}=\frac{z}{d}, w^{*}=\frac{d}{v} w, t^{*}=\frac{v}{d^{2}} t, \Theta^{*}=\frac{\kappa}{\beta v d} \Theta, \Phi^{*}=\frac{(1+\chi) \kappa}{K \beta v d^{2}} \Phi
$$

where $v=\mu / \rho_{0}$ is the kinematic viscosity and $\kappa=k_{1} / \rho_{0} C_{0}$ is the effective thermal diffusivity, we obtain (after dropping the asterisks for simplicity)

$$
\begin{gathered}
{\left[D^{2}-a^{2}-\omega\right]\left(D^{2}-a^{2}\right) W+a^{2}\left[R a_{m} D \Phi-\left(R a+R a_{m}\right) \Theta\right]=0 .} \\
\left(D^{2}-a^{2}-\omega P r\right) \Theta+W=0 . \\
\left(D^{2}-M_{3} a^{2}\right) \Phi-D \Theta=0 .
\end{gathered}
$$

Here, $W, \Theta, \Phi$ are respectively the z-component perturbed amplitudes of velocity, temperature and magnetization term. In addition $D \equiv \mathrm{d} / \mathrm{d} z$ differential operator, $a=\sqrt{l^{2}+m^{2}}$ wave number, $R a=\alpha_{t} g \beta d^{4}$ thermal Rayleigh number, $M_{1}=\mu_{0} K^{2} \beta /(1+\chi) \alpha_{t} \rho_{0} C$ magnetic number, $R a_{m}=R a M_{1}=\mu_{0} K^{2} \beta^{2} d^{4} /(1+\chi) \mu \kappa$ magnetic thermal Rayleigh number, $M_{2}=\mu_{0} T_{0} K^{2} /(1+\chi) \rho_{0} C$ magnetic parameter, $M_{3}=\left(1+M_{0} / H_{0}\right) /(1+\chi)$ magnetization nonlinearity parameter and $\operatorname{Pr}=v / \kappa$ Prandtl number.

We impose the boundary conditions (see Ref. [5] [12] [26]):

$$
\begin{gathered}
W=D W=0, D \Theta=0, \Phi=0 \text { at } z=0 \\
W=D^{2} W+a^{2} M a \Theta=0, D \Theta+B i \Theta=0, D \Phi=0 \text { at } z=1
\end{gathered}
$$

or

$$
W=D^{2} W+M a a^{2} \Theta=0, D \Theta+B i \Theta=0, D \Phi-\Theta=0 \text { at } z=1
$$

where, $M a=\sigma_{T} \Delta T d / \mu \kappa$ the Marangoni number and $B i=h_{t} d / k_{1}$ the Biot number.

\section{Numerical Solution}

The Galerkin method is applied to solve the problem of eigenvalue constituted by Equations (18)-(20) subject to Equations (21)-(23) and accordingly the expanded unknown variables are

$$
\{W, \Theta, \Phi\}(z)=\sum_{m=1}^{n}\left\{A_{m} W_{m}, B_{m} \Theta_{m}, C_{m} \Phi_{m}\right\}(z)
$$

where $A_{m}, B_{m}, C_{m}$ are constants and basis functions $W_{m}, \Theta_{m}, \Phi_{m}$ are trial chosen usually satisfying the considered boundary conditions as follows

$$
W_{m}=\left(z^{3}-5 z^{2} / 2+3 z / 2\right) z^{m}, \Theta_{m}=z(1-z / 2) z^{m}, \Phi_{m}=z^{2}(1-z / 3) z^{m} .
$$

By introducing Equation (25) into Equations (18)-(20), multiplying the resulting equations respectively by $W_{m}, \Theta_{m}$ and $\Phi_{m}$, integrating between $z=0$ and $z=1$ and using Equations (21)-(23) yields

$$
\begin{gathered}
C_{n m} A_{m}+M_{n m} B_{m}+F_{n m} C_{m}=0 . \\
G_{n m} A_{m}+H_{n m} C_{m}=0 . \\
I_{n m} C_{m}+J_{n m} D_{m}=0 .
\end{gathered}
$$

where 


$$
\begin{gathered}
C_{m n}=\left\langle D^{2} W_{m} D^{2} W_{n}\right\rangle+2 a^{2}\left\langle D W_{m} D W_{n}\right\rangle+a^{4}\left\langle W_{m} W_{n}\right\rangle, \\
M_{m n}=-a^{2} R a\left(1+M_{1}\right)\left\langle W_{m} \Theta_{n}\right\rangle+a^{2} M a D W_{m}(1) \Theta_{n}(1) \\
F_{m n}=a^{2} R a M_{1}\left\langle W_{m} D \Phi_{n}\right\rangle \\
G_{m n}=-\left\langle\Theta_{m} W_{n}\right\rangle, \\
H_{m n}=\left\langle a^{2} \Theta_{m} \Theta_{n}+D \Theta_{m} D \Theta_{n}\right\rangle+B i D \Phi_{m}(1) \Theta_{n}(1) \\
I_{m n}=-\left\langle D \Phi_{m} \Theta_{n}\right\rangle \\
J_{m n}=\left\langle a^{2} M_{3} \Phi_{m} \Phi_{n}+D \Phi_{m} D \Phi_{n}\right\rangle
\end{gathered}
$$

with $\langle\cdots\rangle=\int_{0}^{1}(\cdots) \mathrm{d} z$

Equations (26)-(28) may have a solution of non-trivial solution if

$$
\left|\begin{array}{ccc}
C_{n m} & D_{n m} & E_{n m} \\
F_{n m} & G_{n m} & 0 \\
0 & H_{n m} & I_{n m}
\end{array}\right|=0
$$

It would be informative to seem at the results for $m=n=1$ as it gives adequate physical insight into the problem with minimum mathematical computations. For this order, Equation (29) in terms of $M a$ gives the following characteristic equation (after omitting the subscript 1 )

$$
M a=\frac{\eta_{1}+\Omega \eta_{2}}{1260 a^{2}\langle W \Theta\rangle}\left[\frac{70 B i}{\eta_{3}}+\left(a^{2}+\Omega P r\right)\right]+\frac{140 R_{m}\langle W D \varphi\rangle}{\eta_{3}}-2\left(R_{a}+R_{m}\right)\langle W \Theta\rangle(30)
$$

where $\eta_{1}=4536+432 a^{2}+541 a^{4}, \quad \eta_{2}=216+541 a^{2}$ and $\eta_{3}=56+11 M_{3} a^{2}$.

To stability of the system is examined by taking $\Omega=i \omega$ in Equation (30) and the complex quantities have to be clearly yields

$$
\begin{aligned}
M a= & \frac{1}{1260 a^{2}\langle W \Theta\rangle}\left[\eta_{1}\left(\frac{70 B i}{\eta_{3}}+a^{2}\right)-\operatorname{Pr} \eta_{2} \omega^{2}\right] \\
& +\frac{140 R_{m}\langle W D \varphi\rangle}{\eta_{3}}-2\left(R_{a}+R_{m}\right)\langle W \Theta\rangle
\end{aligned}
$$

where

$$
N=\frac{1}{1260 a^{2}\langle W \Theta\rangle}\left[\operatorname{Pr} \eta_{1}+\eta_{2}\left(\frac{70 B i}{\eta_{3}}+a^{2}\right)\right] .
$$

The steady onset (i.e., direct bifurcation) is governed by $\omega=0$ and it occurs at $M a=M a^{s}$, where

$$
M a=\frac{1}{1260 a^{2}\langle W \Theta\rangle}\left[\eta_{1}\left(\frac{70 B i}{\eta_{3}}+a^{2}\right)\right]+\frac{140 R_{m}\langle W D \Phi\rangle}{\eta_{3}}-2\left(R_{m}+R_{a}\right)\langle W \Theta\rangle
$$

\section{Numerical Results and Discussion}

Equation (29) leads to characteristic equation 


$$
f\left(R a, M a, B i, M_{1}, M_{3}, a\right)=0 .
$$

Here we note that the minimum of $R a$ corresponding to $a_{c}$ is to be found that for various physical parameters $M a, B i, M_{1}$ and $M_{3}$. Mathematica 12.0 symbolic algebraic package is applied to compute numerically by Galerkin method for fixing the other parameters with three sets of boundary combinations. The value of $\left(R a_{c}, a_{c}\right)$ obtained here are compared with Sparrow et al. [27]. The results established are in admirable agreement and thus validate the exactness of the numerical technique utilized (see Table 1).

The loci of $M a_{c}$ and $R a_{c}$ with $B i, M_{1}$ and $M_{3}$ are shown in Figures 2(a)-4(a) respectively as well as different magnetic boundaries at the upper surfaces like $D \Phi-\Theta=0$ and $D \Phi=0$ at $z=1$. It is noticeable that, curves are slightly convex and there is a strong coupling between $M a_{c}$ and $R a_{c}$. If the magnetic force is leading, then the surface tension becomes insignificant and vice-versa. A review of Figure 2(a), further reveals that with increase in $B i$ it delays the FTC. This may be attributed to fact that with increasing $B i$, the free surface gets deviated from good conductor of heat and there is an increase in $M a_{c}$ and $R a_{c}$. Also $D \Phi-\Theta=0$ surfaces offer more stabilizing effect compared to $D \Phi=0$ against FTC. Figure 2(b) illustrates that increasing in $a_{c}$ as $B i$ increases, hence its effect is to diminish the size of convection cells.

In Figure 3(a), $R a_{c}$ and $M a_{c}$ presented with $M_{1}$ when $M_{3}=1$ and $B i=2$. This is expected that an increase in $M_{1}$ is to decrease $M a_{c}$ and $R a_{c}$, thus leads to a more unstable system due to an increase in magnetic force. Moreover, it is remarkable $M a_{c}$ and $R a_{c}$ are diminishes as $M_{1}$ increases. From Figure 3(b), increase $M_{1}$ is to increase $a_{c}$, thus leading to diminish the convection cell size..

The effect of increase in $M_{3}$ is shown in Figure 4(a) for $B i=M_{1}=2$ and it is Table 1. Comparison of $\left(R a_{c}, a_{c}\right)$ with $B i$ for $M a=M_{1}=0$.

\begin{tabular}{ccccc}
\hline \multirow{2}{*}{$B i$} & \multicolumn{2}{c}{ Sparrow et al. $[27]$} & \multicolumn{2}{c}{ Present study } \\
\cline { 2 - 5 } & $R a_{c}$ & $a_{c}$ & $R a_{c}$ & $a_{c}$ \\
\hline 0 & 320.000 & 0.00 & 320.000 & $-2.641 \times 10^{-9}$ \\
0.01 & 338.905 & 0.58 & 338.904 & 0.5831 \\
0.03 & 353.176 & 0.76 & 353.158 & 0.7624 \\
0.1 & 381.665 & 1.015 & 381.665 & 1.0151 \\
0.3 & 428.290 & 1.03 & 428.290 & 1.2992 \\
1 & 513.792 & 1.64 & 513.790 & 1.6438 \\
3 & 619.666 & 1.92 & 619.666 & 1.9211 \\
30 & 780.240 & 2.18 & 780.237 & 2.1760 \\
100 & 804.973 & 2.20 & 804.972 & 2.2029 \\
$\infty$ & 816.748 & 2.21 & 816.744 & 2.2147 \\
\hline
\end{tabular}



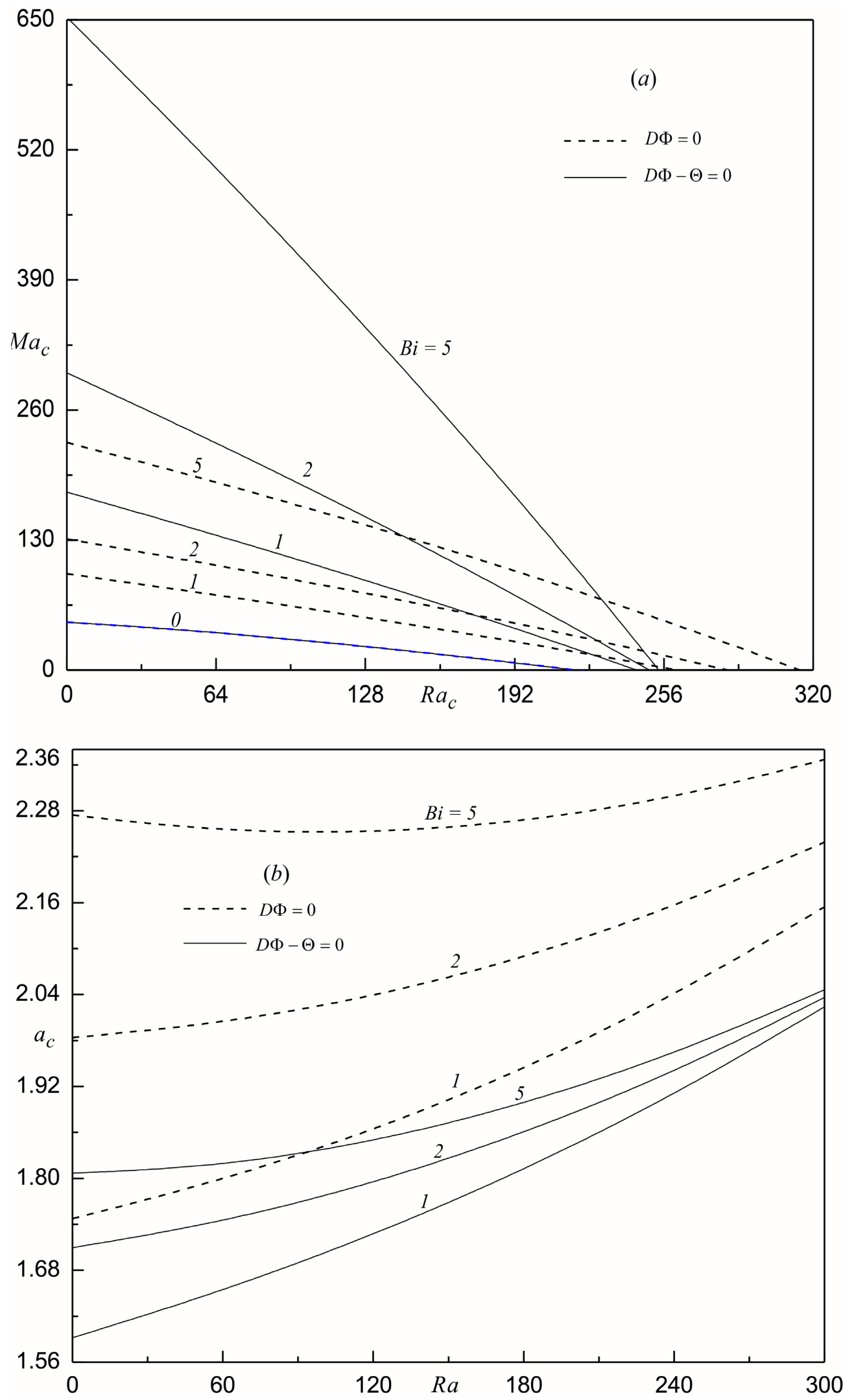

Figure 2. (a) $M a_{c}$ versus $R a_{c}$ with $B i$ when $M_{3}=1$ and $M_{1}=2$; (b) $a_{c}$ versus $R a_{c}$ with $B i$ when $M_{3}=1$ and $M_{1}=2$. 

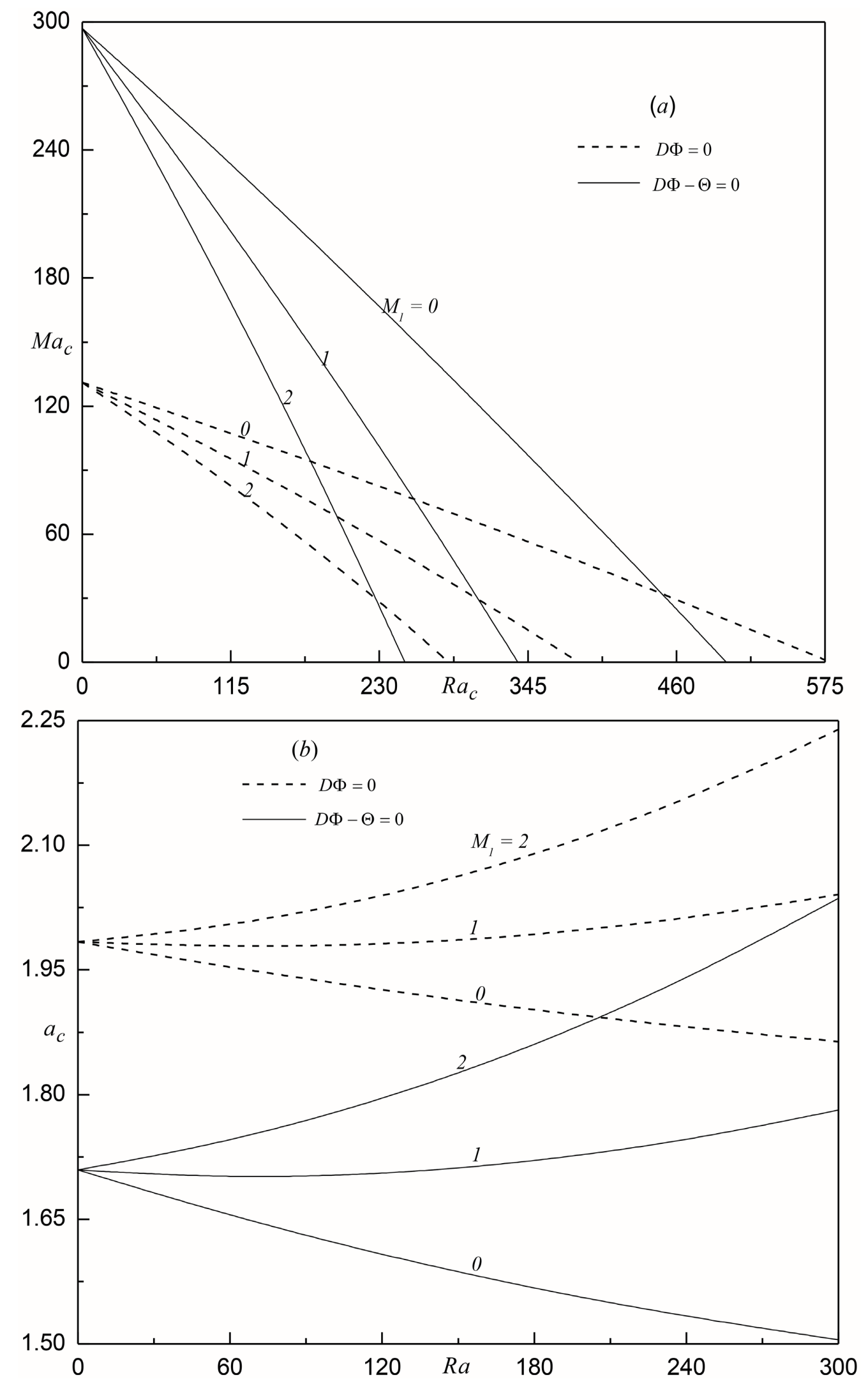

Figure 3. (a) $M a_{c}$ versus $R a_{c}$ with $M_{1}$ when $M_{3}=1$ and $B i=2$; (b) $a_{c}$ versus $R a$ with $M_{1}$ when $M_{3}=1$ and $B i=2$. 

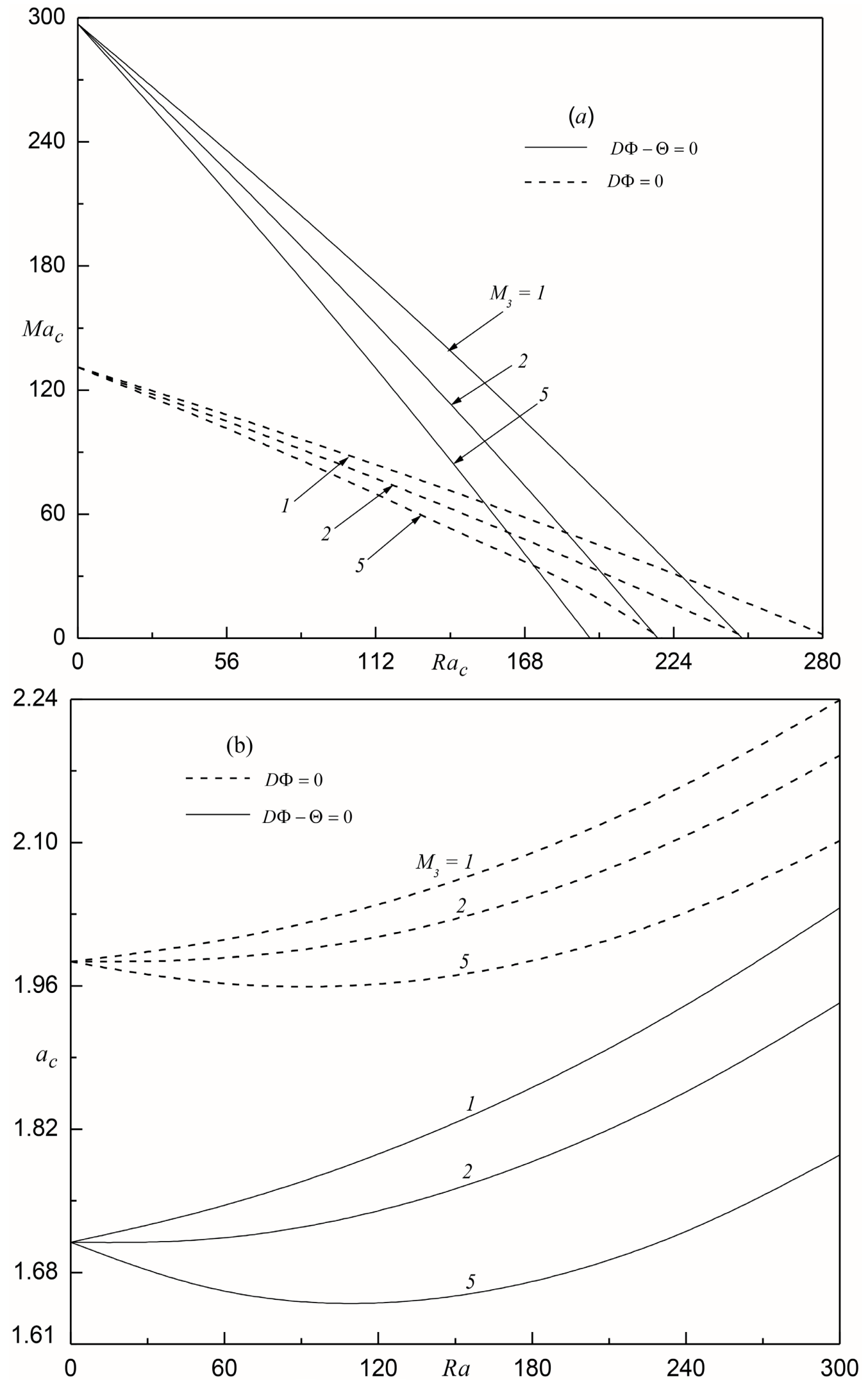

Figure 4. (a) $M a_{c}$ versus $R a_{c}$ with $M_{3}$ when $M_{1}=2$ and $B i=2$; (b) $a_{c}$ versus $R a_{c}$ with $M_{3}$ when $M_{1}=2$ and $B i=2$. 
observed the stability parameters $R a_{c}$ and $M a_{c}$ decreases as increasing $M_{3}$, thus the mechanism of magnetization non-linearity parameter has a destabilizing effect on the system. Nonetheless, $R a_{c}$ and $M a_{c}$ are found to be independent of $M_{3}$ for $B i=0$. While the value of $a_{c}$ decreases as increasing in $M_{3}$ and thus the effect is to enlarge size of convection cells.

\section{Conclusions}

The influence of general boundary conditions on buoyancy and surface tensiondriven FTC in a FF layer is investigated numerically Galrkin technique based on weighted residual technique. The following conclusions were resulting:

- The initiation of FTC is inhibited with increasing Biot number $B i$.

- The magnetic parameter $M_{1}$ and fluid magnetization non-linearity parameter $M_{3}$ hasten the FTC.

- The magnetic bounding surfaces $D \Phi-\Theta$ offer more stabilizing while $D \Phi$ surfaces offer least stable effects against FTC. i.e. $\left(R a_{c} \text { or } M a_{c}\right)_{D \Phi}<\left(R a_{c} \text { or } M a_{c}\right)_{D \Phi-\Theta}$.

- The critical value $\left(a_{c}\right)$ for $D \Phi$ is always higher than those of remaining boundaries. i.e. $\left(a_{c}\right)_{D \Phi-\Theta}<\left(a_{c}\right)_{D \Phi}$.

\section{Conflicts of Interest}

The authors declare no conflicts of interest regarding the publication of this paper.

\section{References}

[1] Rosensweig, R.E. (1985) Ferrohydrodynamics. Cambridge University Press, Cambridge.

[2] Bashtovoy, V.G., Berkovsky, B.N. and Vislovich, A.N. (1988) Introduction to Thermo Mechanics of Magnetic Fluids. Springer, Berlin, Heidelberg.

[3] Berkovsky, B.M., Medvedev, V.F. and Krakov, M.S. (1993) Magnetic Fluids, Engineering Applications. Oxford University Press, Oxford.

[4] Odenbach, S. (2003) Magnetic Fluids-Suspensions of Magnetic Dipoles and Their Mantic Controls. Journal of Physics. Condensed Matter, 15, S1497-S1508. https://doi.org/10.1088/0953-8984/15/15/312

[5] Finlayson, B.A. (1972) Method of Weighted Residuals and Variational Principles. Academic Press, Cambridge.

[6] Qin, Y. and Kaloni, P.N. (1994) Nonlinear Stability Problem of a Ferromagnetic Fluid with Surface Tension Effect. European Journal of Mechanics B: Fluids, 13, 305-321.

[7] Hennenberg, M., Weyssow, B., Slavtchev, S. and Legros, J.C. (2001) Coupling between Marangoni and Rosensweig Instabilities. Part I: The Transfer Wave. European Physics Journal of Applied Physics, 16, 217-229.

https://doi.org/10.1051/epjap:2001212

[8] Shivakumara, I.S., Rudraiah, N. and Nanjundappa, C.E. (2002) The Effect of Different forms of Basic Temperature Gradients on the Onset of Ferroconvection Driven by Combined Surface Tension and Buoyancy Forces. Indian Journal of Pure 
Applied Physics, 40, 95-106.

[9] Shivakumara, I.S. and Nanjundappa, C.E. (2006) Marngoni Ferroconvection with Different Initial Temperature Gradients. Journal of Energy and Heat Mass Transfer, 28, 1-45.

[10] Nanjundappa, C.E. and Shivakumara, I.S. (2008) Effect of Velocity and Temperature Boundary Conditions on Convective Instability in a Ferrofluid Layer. ASME Journal of Heat Transfer, 130, Article ID: 104502. https://doi.org/10.1115/1.2952742

[11] Shivakumara, I.S., Nanjundappa, C.E. and Chavaraddi, K.B. (2009) Darcy-BenardMarangoni Convection in Porous Media. International Journal of Heat and Mass Transfer, 52, 2815-2823. https://doi.org/10.1016/j.ijheatmasstransfer.2008.09.038

[12] Nanjundappa, C.E., Shivakumara, I.S. and Arunkumar, R. (2010) Bénard-Marangoni Ferroconvection with Magnetic Field Dependent Viscosity. Journal of Magnetism and Magnetic Materials, 322, 2256-2263. https://doi.org/10.1016/j.jmmm.2010.02.021

[13] Nanjundappa, C.E., Shivakumara, I.S. and Savitha, B. (2014) Onset of Bénard-Marangoni Rroconvection with a Convective Surface Boundary Condition: The Effects of Cubic Temperature Profile and MFD Viscosity. International Communications in Heat and Mass Transfer, 51, 39-44. https://doi.org/10.1016/j.icheatmasstransfer.2013.11.010

[14] Nanjundappa, C.E., Shivakumara, I.S. and Arunkumar, R. (2013) Onset of Marangoni-Bénard Ferroconvection with Temperature Dependent Viscosity. Microgravity Science and Technology, 25, 103-112. https://doi.org/10.1007/s12217-012-9330-9

[15] Nanjundappa, C.E., Prakash, H.N., Shivakumara, I.S. and Lee, J. (2014) Effect of Temperature Dependent Viscosity on the Onset of Bénard-Marangoni Ferroconvection. International Communications in Heat and Mass Transfer, 51, 25-30. https://doi.org/10.1016/j.icheatmasstransfer.2014.01.010

[16] Nanjundappa, C.E., Shivakumara, I.S. and Arunkumar, R. (2011) Onset of BénardMarangoni Ferroconvection with Internal Heat Generation. Microgravity Science and Technology, 23, 29-39. https://doi.org/10.1007/s12217-010-9218-5

[17] Nanjundappa, C.E., Shivakumara, I.S. and Srikumar, K. (2013) On the Penetrative Bénard-Marangoni Convection in a Ferromagnetic Fluid Layer. Aerospace Science and Technology, 27, 57-66. https://doi.org/10.1016/j.ast.2012.06.007

[18] Shivakumara, I.S. and Nanjundappa, C.E. (2006) Effects of Coriolis Force And Different Basic Temperature Gradients On Marangoni Ferroconvection. Acta Mechanica, 182, 113-124. https://doi.org/10.1007/s00707-005-0296-1

[19] Nanjundappa, C.E., Shivakumara, I.S. and Lee, J. (2015) Effect of Coriolis Force on Bénard-Marangoni Convection in a Rotating Ferrofluid Layer with MFD Viscosity, Microgravity Science and Technology, 27, 27-37.

https://doi.org/10.1007/s12217-014-9410-0

[20] Shivakumara, I.S., Lee, J. and Nanjundappa, C.E. (2012) Onset of Thermogravitational Convection in a Ferrofluid Layer with Temperature Dependent Viscosity. Journal of Heat Transfer, 134, Article ID: 012501. https://doi.org/10.1115/1.4004758

[21] Nanjundappa, C.E., Ravisha, M., Lee, J. and Shivakumara, I.S. (2011) Penetrative Ferroconvection in a Porous Layer. Acta Mechanica, 216, 243-257.

https://doi.org/10.1007/s00707-010-0367-9

[22] Nanjundappa, C.E., Shivakumara, I.S., Lee, J. and Ravisha, M. (2011) Effect Of Internal Heat Generation on the Onset of Brinkman-Bénard Convection in a Ferrofluid Saturated Porous Layer. International Journal of Thermal Sciences, 50, 160-168. https://doi.org/10.1016/j.ijthermalsci.2010.10.003 
[23] Nanjundappa, C.E., Shivakumara, I.S. and Prakash, H.N. (2012) Penetrative Ferroconvection via Internal Heating in a Saturated Porous Layer with Constant Heat Flux at the Lower Boundary. Journal of Magnetism and Magnetic Materials, 324, 1670-1678. https://doi.org/10.1016/j.jmmm.2011.11.057

[24] Nanjundappa, C.E., Pavithra, A. and Shivakuamara, I.S. (2021) Effect of Dusty Particles on Darcy-Brinkman Gravity-Driven Ferro-Thermal-Convection in a Ferrofluid Saturated Porous Layer with Internal Heat Source: Influence of Boundaries, International Journal of Applied Computational Mathematics, 7, Article No. 21. https://doi.org/10.1007/s40819-020-00948-6

[25] Savitha, Y.L., Nanjundappa, C.E. and Shivakumara, I.S. (2021) Penetrative Brinkman Ferroconvection via Internal Heating in High Porosity Anisotropic Porous Layer: Influence of Boundaries. Heliyon, 7, Article ID: e06153.

https://doi.org/10.1016/j.heliyon.2021.e06153

[26] Gotoh, K. and Yamada, M. (1982) Thermal Convection in a Horizontal Layer of Magnetic Fluids. Journal of Physical Society of Japan, 51, 3042-3048.

https://doi.org/10.1143/JPSI.51.3042

[27] Sparrow, E.M., Goldstein, R.J. and Jonsson, U.K. (1964) Thermal Instability in a Horizontal Fluid Layer: Effect of Boundary Conditions and Non-Linear Temperature Profiles. Journal of Fluid Mechanics, 18, 513-528.

https://doi.org/10.1017/S0022112064000386 